\title{
Numerical Integration over the Spherical Shell
}

1. Introduction. Numerical integration over three-dimensional regions has received relatively little attention. The papers listed in the bibliography give the work on this subject known to the author. With regards the sphere, Hammer and Wymore [6], have given formulas with specific polynomial accuracy. This paper gives a formula for arbitrarily high polynomial accuracy and generalizes the region to the spherical shell.

2. Type of formula. The type of formula considered is a weighted sum of integrand values

$$
\iiint f(x, y, z) d x d y d z \doteq \sum_{i=1}^{n} a_{i} f\left(x_{i}, y_{i}, z_{i}\right),
$$

where the dot over the equals sign is meant to signify that in general the right hand side will only be an approximation to the left hand side. The region under consideration is the spherical shell of inner radius $R$ and outer radius one. By a theorem of Hammer and Wymore [7], a formula over any region which is an affine transformation of this spherical shell can be easily obtained. The criterion used to determine the weights, $a_{i}$, and the points, $\left(x_{i}, y_{i}, z_{i}\right)$, is that the formula shall be exact whenever the integrand function is a polynomial in $x, y$, and $z$ of degree at most $s$.

A numerical integration formula, whether it be in one, two, or more variables, is said to have accuracy $s$ provided the formula is exact whenever the integrand function is a polynomial of degree $s$ or less, and provided there exists at least one polynomial of degree $s+1$ for which the formula is not exact.

3. Numerical integration over the spherical shell. We first transform the problem to one in spherical coordinates. Under the transformation

$$
\begin{aligned}
& x=r \sin \phi \cos \theta \\
& y=r \sin \phi \sin \theta \\
& z=r \cos \phi
\end{aligned}
$$

the integral $\iiint f(x, y, z) d x d y d z$ over the spherical shell with inner radius $R$ and outer radius one can be written as

$$
\int_{R}^{1} \int_{0}^{\pi} \int_{0}^{2 \pi} r^{2} \sin \phi F(\theta, \phi, r) d \theta d \phi d r
$$

where $F(\theta, \phi, r) \equiv f(r \sin \phi \cos \theta, r \sin \phi \sin \theta, r \cos \phi)$. Since any formula for the integral in rectangular form is to be exact whenever the integrand function is a polynomial of degree at most $s$ in $x, y$, and $z$, any formula for the integral in spherical form must be exact whenever $F(\theta, \phi, r)$ is a polynomial of degree at most $\sin r \sin \phi \cos \theta, r \sin \phi \sin \theta$, and $r \cos \phi$. The type of formula we attempt to find is

(1) $I=\int_{R}^{1} \int_{0}^{\pi} \int_{0}^{2 \pi} r^{2} \sin \phi F(\theta, \phi, r) d \theta d \phi d r \doteq \sum_{i} \sum_{j} \sum_{k} D_{i j k} F\left(\theta_{i}, \phi_{j}, r_{k}\right)$, 
which is a weighted sum of integrand values where the limits on $i, j$, and $k$ are to be determined. We now state the main result of this paper in the following theorem.

THEOREM. If it be required that

(a) formula (1) have accuracy $s=4 m+3, m=0,1,2, \cdots$, in $r \sin \phi \cos \theta$, $r \sin \phi \sin \theta$, and $r \cos \phi$,

(b) the evaluation points be taken at all intersection points of the planes $\theta=\theta_{i}$, the planes $\phi=\phi_{j}$, and the spheres $r=r_{k}$,

(c) the evaluation points under this configuration be minimum in number,

then it is both necessary and sufficient for the existence of a unique set of real weights $D_{i j k}$ that

(a) $\theta_{i}=2 \pi_{i} /(s+1), i=1,2, \cdots, s+1$,

(b) the $\cos \phi_{j}$ be the $2 m+2$ zeros of the Legendre polynomial $P_{2 m+2}$ of degree $2 m+2$, orthogonalized on $[-1,1]$,

(c) the $r_{k}^{2}$ be the $m+1$ zeros of the polynomial in $r^{2}$ of degree $m+1, Q_{m+1}\left(r^{2}\right)$, where $\int_{R}^{1} r Q_{m+1}\left(r^{2}\right) T_{m}\left(r^{2}\right) d r^{2}=2 \int_{R}^{1} r^{2} Q_{m+1}\left(r^{2}\right) T_{m}\left(r^{2}\right) d r=0$, and where $T_{m}\left(r^{2}\right)$ is an arbitrary polynomial in $r^{2}$ of degree $m$ or less.

The unique weights $D_{i j k}$ are equal to $A_{i} B_{j} C_{k}$ where

$$
\begin{gathered}
A_{i}=\frac{2 \pi}{s+1}, \quad i=1,2, \cdots, s+1, \\
B_{i}=\frac{1}{P_{2 m+2}^{\prime}\left(y_{j}\right)} \int_{-1}^{1} \frac{P_{2 m+2}(y)}{y-y_{j}} d y, j=1,2, \cdots, 2 m+2, \\
C_{k}=\frac{1}{Q_{m+1}^{\prime}\left(r_{k}^{2}\right)} \int_{R}^{1} \frac{r^{2} Q_{m+1}\left(r^{2}\right)}{r^{2}-r_{k}^{2}} d r, k=1,2, \cdots, m+1,
\end{gathered}
$$

or

$$
C_{k}=\frac{1}{2 Q_{m+1}^{\prime}\left(t_{k}\right)} \int_{R^{2}}^{1} \frac{\sqrt{ } t Q_{m+1}(t)}{t-t_{k}} d t, \text { where } t=r^{2} .
$$

In the first expression for $C_{k}$, the notation $Q_{m+1}^{\prime}\left(r_{k}^{2}\right)$ indicates a derivative with respect to $r^{2}$.

Proof. Because of the arrangement of the points we have chosen, we write the left hand part of (1) as

Now define

$$
I=\int_{R}^{1} r^{2} \int_{0}^{\pi} \sin \phi \int_{0}^{2 \pi} F(\theta, \phi, r) d \theta d \phi d r .
$$

which gives

$$
\begin{aligned}
& H(\phi, r)=\int_{0}^{2 \pi} F(\theta, \phi, r) d \theta \\
& G(r)=\int_{0}^{\pi} \sin \phi H(\phi, r) d \phi
\end{aligned}
$$

$$
I=\int_{R}^{1} r^{2} G(r) d r
$$


We now look for three formulas, namely

$$
\begin{gathered}
H(\phi, r)=\int_{0}^{2 \pi} F(\theta, \phi, r) d \theta \doteq \sum_{i=1}^{p} A_{i} F\left(\theta_{i}, \phi, r\right), \\
G(r)=\int_{0}^{\pi} \sin \phi H(\phi, r) d \phi \doteq \sum_{i=1}^{q} B_{j} H\left(\phi_{j}, r\right), \\
I=\int_{R}^{1} r^{2} G(r) d r \doteq \sum_{k=1}^{v} C_{k} G\left(r_{k}\right) .
\end{gathered}
$$

When these three formulas have been obtained, we will combine them to obtain formula (1) with $D_{i j k}=A_{i} B_{j} C_{k}$.

Formula (2)

$$
H(\phi, r)=\int_{0}^{2 \pi} F(\theta, \phi, r) d \theta \doteq \sum_{i=1}^{p} A_{i} F\left(\theta_{i}, \phi, r\right) .
$$

The number $p$ is to be determined, and this formula must be exact whenever $F(\theta, \phi, r)$ is a polynomial of degree $s$ in $r \sin \phi \cos \theta, r \sin \phi \sin \theta$, and $r \cos \phi$. Since $r$ and $\phi$ are parameters here, we can require formula (2) to be exact whenever $F$ is a polynomial of degree $s$ in $\cos \theta$ and $\sin \theta$, and hence whenever $F=1$, $\cos \theta, \sin \theta, \cos 2 \theta, \sin 2 \theta, \cdots, \cos s \theta, \sin s \theta$. This leads to a system of $2 s+1$ equations

$$
\begin{gathered}
A_{1}+A_{2}+\cdots+A_{p}=\int_{0}^{2 \pi} 1 d \theta=2 \pi \\
A_{1} \cos t \theta_{1}+A_{2} \cos t \theta_{2}+\cdots+A_{p} \cos t \theta_{p}=\int_{0}^{2 \pi} \cos t \theta d \theta=0 \\
A_{1} \sin t \theta_{1}+A_{2} \sin t \theta_{2}+\cdots+A_{p} \sin t \theta_{p}=\int_{0}^{2 \pi} \sin t \theta d \theta=0
\end{gathered}
$$

for $t=1,2, \cdots, s$.

One solution to this system (a sufficient condition that formula (2) have accuracy $s$ with minimum $p$ ) is given by

$$
\begin{gathered}
p=s+1 \\
A_{i}=\frac{2 \pi}{s+1}, \quad i=1,2, \cdots, s+1 \\
\theta_{i}=\frac{2 \pi i}{s+1}, \quad i=1,2, \cdots, s+1 .
\end{gathered}
$$

The requirement that the $A_{i}$ be real leads to the conclusion that this is the only solution up to a rotation. (For the idea which led to this result, I am grateful to Professor Fritz Herzog.) The above is therefore a necessary and sufficient condition that formula (2) have accuracy $s$ in $r \sin \phi \cos \theta, r \sin \phi \sin \theta$, and $r \cos \phi$.

Formula (3)

$$
G(r)=\int_{0}^{\pi} \sin \phi H(\phi, r) d \phi=\sum_{j=1}^{q} B_{j} H\left(\phi_{i}, r\right)
$$

The number $q$ has to be determined, and this formula, like (2), must be exact whenever $F(\theta, \phi, r)$ is a polynomial of degree $s$ in $r \sin \phi \cos \theta, r \sin \phi \sin \theta$, and $r \cos \phi$. Considering the definition of $H(\phi, r)$, formula (3) must therefore be exact whenever $H(\phi, r)$ is a polynomial of degree $s$ in $\cos \phi$. This leads to a system of 
$s+1$ equations

$$
B_{1} \cos { }^{t} \phi_{1}+B_{2} \cos { }^{t} \phi_{2}+\cdots+B_{q} \cos { }^{t} \phi_{q}=\int_{0}^{\pi} \sin \phi \cos { }^{t} \phi d \phi
$$

for $t=0,1,2, \cdots, s$. Putting $\cos \phi=y$, these equations become

$$
B_{1} y_{1}^{t}+B_{2} y_{2}^{t}+\cdots+B_{q} y_{q}^{t}=\int_{-1}^{1} y^{t} d y \text {. }
$$

The unique solution to this system for minimum $q$ has been given by Gauss [4] and is

$$
\begin{aligned}
q & =\frac{s+1}{2}=2 m+2, \\
y_{i} & =\cos \phi_{i}=\text { the } q=2 m+2 \text { zeros of the Legendre polynomial } P_{2 m+2} \text { of } \\
& \text { degree } 2 m+2, \text { orthogonalized on }[-1,1] . \\
B_{i} & =\frac{1}{P_{2 m+2}^{\prime}\left(y_{j}\right)} \int_{-1}^{1} \frac{P_{2 m+2}(y)}{y-y_{j}} d y,
\end{aligned}
$$

where $P^{\prime}$ means the derivative of $P$ with respect to $y$.

Formula (4)

$$
I=\int_{R}^{1} r^{2} G(r) d r \doteq \sum_{k=1}^{v} C_{k} G\left(r_{k}\right)
$$

Again, the number $v$ has to be determined, and this formula, like (2) and (3), must be exact whenever $F(\theta, \phi, r)$ is a polynomial of degree $s$ in $r \sin \phi \cos \theta, r \sin \phi$ $\sin \theta$, and $r \cos \phi$. From the definition of $G(r)$, formula (4) must be exact whenever $G(r)$ is a polynomial in $r^{2}$ of degree $(s-1) / 2=2 m+1$. Putting $g\left(r^{2}\right)=$ $G(r)$, formula (4) can be written

$$
2 I=\int_{R}^{1} r g\left(r^{2}\right) d r^{2} \doteq \sum_{k=1}^{v} 2 C_{k} g\left(r_{k}^{2}\right)
$$

The unique solution for the $C_{k}$ and the $r_{k}$ for minimum $v$ is given by Szegö [11] and is

$$
\begin{aligned}
v= & m+1 \\
r_{k}^{2}= & \text { the } m+1 \text { zeros of the polynomial in } r^{2} \text { of degree } m+1, Q_{m+1}\left(r^{2}\right), \\
& \text { where } \int_{R}^{1} r Q_{m+1}\left(r^{2}\right) T_{m}\left(r^{2}\right) d r^{2}=2 \int_{R}^{1} r^{2} Q_{m+1}\left(r^{2}\right) T_{m}\left(r^{2}\right) d r=0, \quad \text { and }
\end{aligned}
$$

where $T_{m}\left(r^{2}\right)$ is an arbitrary polynomial in $r^{2}$ of degree $m$ or less,

$$
C_{k}=\frac{1}{Q_{m+1}^{\prime}\left(r_{k}^{2}\right)} \int_{\mathrm{R}}^{1} \frac{r^{2} Q_{m+1}\left(r^{2}\right)}{r^{2}-r_{k}^{2}} d r
$$

where $Q^{\prime}$ means the derivative of $Q$ with respect to $r^{2}$. This completes the proof of the theorem.

Several corollaries are immediate.

Corollary. The weights $D_{i j k}=A_{i} B_{j} C_{k}$ are positive, and their sum is the volume of the spherical shell.

COROLLARY. All evaluation points of the formula lie between the inner and outer sphere of the spherical shell.

CoROLlaRY. The number of evaluation points is $8(m+1)^{3}$. 
Although $8(m+1)^{3}$ is the minimum number of points for the point configuration we have chosen, it is not necessarily the minimum number for other point configurations. For example, the formula developed by Hammer and Wymore [7] for 7 th degree accuracy utilizes only 27 points, whereas the formula developed here uses 64 points for the same accuracy. The formulas presented here, however, give formulas of arbitrarily high accuracy. (By removing the restriction that $s$ be of the form $4 m+3$, which can be done, it is possible in some cases to reduce the number of points below the number specified by $8(m+1)^{3}$.

4. The weights $\boldsymbol{C}_{k}$ and the zeros of $\boldsymbol{Q}_{m+1}\left(\boldsymbol{r}^{2}\right)$. We list below, for $m=0$ and for $m=1$, and for four values of the inner radius $R$, the polynomials $Q_{m+1}\left(r^{2}\right)$, their zeros, and the weights $C_{k}$. (Decimal numbers are truncated, not rounded.)

$$
\begin{aligned}
& m=0 \\
& R=0 \quad Q_{1}\left(r^{2}\right)=r^{2}-\frac{3}{5} \\
& r_{1}^{2}=.6000000000 \quad C_{1}=.3333333333 \\
& R=\frac{1}{4} \quad Q_{1}\left(r^{2}\right)=r^{2}-\frac{341}{560} \\
& r_{1}^{2}=.6089285714 \quad C_{1}=.3281250000 \\
& R=\frac{1}{2} \quad Q_{1}\left(r^{2}\right)=r^{2}-\frac{93}{140} \\
& r_{1}{ }^{2}=.664285714 \quad C_{1}=.2916666666 \\
& R=\frac{3}{4} \quad Q_{1}\left(r^{2}\right)=r^{2}-\frac{2343}{2960} \\
& r_{1}^{2}=.7915540540 \quad C_{1}=.1927083333 \\
& m=1 \\
& R=0 \quad Q_{2}\left(r^{2}\right)=r^{4}-\frac{10}{9} r^{2}+\frac{5}{21} \\
& r_{1}^{2}=.2899491979 \quad C_{1}=.1387779991 \\
& r_{2}^{2}=.8211619131 \quad C_{1}=.1945553342 \\
& R=\frac{1}{4} \quad Q_{2}\left(r^{2}\right)=r^{4}-\frac{107,605}{94,472} r^{2}+\frac{5,464,615}{21,161,728} \\
& r_{1}^{2}=.312393379 \quad C_{1}=.138908126 \\
& r_{2}^{2}=.826621355 \quad C_{2}=.189216873 \\
& R=\frac{1}{2} \quad Q_{2}\left(r^{2}\right)=r^{4}-\frac{5905}{4599} r^{2}+\frac{63,005}{171,696} \\
& r_{1}{ }^{2}=.429405421 \quad C_{1}=.130536459 \\
& r_{2}^{2}=.854569355 \quad C_{2}=.161130205 \\
& R=\frac{3}{4} \quad Q_{2}\left(r^{2}\right)=r^{4}-\frac{2,046,079,840}{1,302,513,408} r^{2}+\frac{782,901,015}{1,302,513,408} \\
& r_{1}{ }^{2}=.6595819 \quad C_{1}=.0916695 \\
& r_{2}{ }^{2}=.9112885 \quad C_{2}=.1010387
\end{aligned}
$$

Electric Boat Division

William H. Peirce

Groton, Connecticut 
1. P. ApPELl, "Sur une classe de polynômes à deux variables et le calcul approché des intégrales doubles," Annales de la Faculté des Sciences de Toulouse, v. 4, 1890, p. H.1-H.20.

2. C. Birindelli, "Su nuove formule interpolatorie del Picone per funzioni in piu variabili e loro contributo al calcolo numerico degli integrali multipli," Compositio Mathematica, v. 10, 1952, p. 117-167.

3. W. BURnside, "An approximate quadrature formula," Messenger of Math., v. 37, 1908, p. $166-167$.

4. C. F. GAuss, "Methodus nova integralium valores per approximationem inveniendi," Werke, Gö̀ttingen, v. 3, 1866, p. 163-196.

5. P. C. Hammer, O. J. Marlowe, \& A. H. Stroud, "Numerical integration over simplexes and cones," $M T A C$, v. 10, 1956, p. 130-137.

6. P. C. Hammer \& Arthur H. Stroud, "Numerical integration over simplexes," $M T A C$, v. 10, 1956, p. 137-139.

7. Preston C. Hammer \& A. Wayne Wymore, "Numerical evaluation of multiple integrals I," $M T A C$, v. 11,1957, p. 59-67.

8. J. C. Maxwell, "On approximate multiple integration between limits by summation," Camb. Phil. Soc., Proc., v. 3, 1877, p. 39-47.

9. M. Picone, "Vedute generali sull' interpolazione e qualche loro conseguenza," Annali della Scuola Normale Superiore di Pisa, Ser. III, v. 5, 1951, p. 193-244.

10. Michael Sadowsky, "A formula for approximate computation of a triple integral," Amer. Math. Mon., v. 47, 1940, p. 539-543.

11. G. Szegö, Orthogonal Polynomials, Amer. Math. Soc. Colloquium Publication, v. 23, New York, 1939.

12. G. W. TyLER, "Numerical integration of functions of several variables," Can. J. Math., v. 5,1953 , p. $393-412$.

13. Richard von Mises, "Numerische Berechnung mehrdimensionaler Integrale," $Z$. Angew. Math. Mech., v. 34, 1954, p. 201-210.

\section{On a Generalization of the Prime Pair Problem}

1. Introduction. One of the many unsolved problems in the theory of prime numbers concerns the celebrated conjecture by A. de Polignac [1] about primes which differ by two. Prime pairs (or twin primes) are generally believed to constitute an infinite set but to date no one has succeeded in proving or disproving this conjecture.

It was established by Brun [2] that the sum of the reciprocals of all prime pairs is bounded but whether this boundedness is due to the finiteness of the set or to the "thinness" of an infinite set has not been determined.

2. Prime n-tuples. In generalizing from prime pairs to prime $n$-tuples we must first define what is meant by a prime $n$-tuple.

Definition: a prime $n$-tuple is a set of $n$ odd primes $p_{1}, p_{2}, \cdots p_{n}$ such that the difference $\Delta n=p_{n}-p_{1}$ is a non-trivial minimum.

The meaning of "non-trivial" will now be explained. For $n=3$ we can exhibit a set of three consecutive odd primes such that $\Delta 3=4$ (i.e., $3,5,7$ ). We consider this a trivial minimum, however, because (with one exception) in every set of three consecutive odd integers one of the integers in the set is composite since it is divisible by 3 . The single exception to this rule is the case where the number divisible by 3 is the number 3 itself. Generally, we consider a set of $n$ consecutive primes a trivial prime $n$-tuple if one of the primes of the set is always a factor of one of the terms of any sequence of consecutive odd integers having the same value for $\Delta n$. 